Research Article

\title{
On the Inverse EEG Problem for a 1D Current Distribution
}

\author{
George Dassios, George Fragoyiannis, and Konstantia Satrazemi \\ Department of Chemical Engineering, University of Patras and ICE/HT-FORTH, Patras, Greece \\ Correspondence should be addressed to George Dassios; gdassios@otenet.gr
}

Received 27 December 2013; Revised 16 May 2014; Accepted 22 May 2014; Published 19 June 2014

Academic Editor: Shan Zhao

Copyright (C) 2014 George Dassios et al. This is an open access article distributed under the Creative Commons Attribution License, which permits unrestricted use, distribution, and reproduction in any medium, provided the original work is properly cited.

\begin{abstract}
Albanese and Monk (2006) have shown that, it is impossible to recover the support of a three-dimensional current distribution within a conducting medium from the knowledge of the electric potential outside the conductor. On the other hand, it is possible to obtain the support of a current which lives in a subspace of dimension lower than three. In the present work, we actually demonstrate this possibility by assuming a one-dimensional current distribution supported on a small line segment having arbitrary location and orientation within a uniform spherical conductor. The immediate representation of this problem refers to the inverse problem of electroencephalography (EEG) with a linear current distribution and the spherical model of the brain-head system. It is shown that the support is identified through the solution of a nonlinear algebraic system which is investigated thoroughly. Numerical tests show that this system has exactly one real solution. Exact solutions are analytically obtained for a couple of special cases.
\end{abstract}

\section{Introduction}

Electroencephalography (EEG) has a history of almost 90 years [1]. It is actually an imaging modality that associates the electric potential that is generated on the surface of the head with the electric neuronal activity in the interior of the head. The brain is modeled as a conductive material and therefore, any elementary current, running in the neurons, generates a secondary induction current in the whole of the conductive brain. Hence, the measurements recorded on the surface of the head involve not only the effects of the primary neuronal current but those of the induction current as well. In some sense, the conductivity of the brain "hides" the primary electric activity. Then, the principal problem of electroencephalography is to strip the secondary effects of the induction current from the measured data, in order to identify the actual neuronal current.

From Maxwell's equations we know that the electric activity of a medium cannot be isolated from the corresponding magnetic one and the coupled field is what we call electromagnetic wave field. However, if the natural wavelength is larger than the characteristic dimension of the medium, then the time-derivative terms of the electric and the magnetic fields in Maxwell's equations can be omitted and the resulting theory is known as the Quasi-Static Theory of Electromagnetism [2]. In the case where the conductive medium is the brain tissue Plonsey and Heppner [3] have calculated the wavelength of the brain to be [4] approximately equal to $400 \mathrm{~m}$. Since the half of this length is much larger than the characteristic dimension of the brain-head system it follows that the Quasi-Static theory is well justified as the appropriate theory for the investigation of the electric and magnetic brain activity.

As it is the case with most of real-life problems, electroencephalography involves a forward and an inverse problem. In the forward EEG problem, the neuronal current is given and we seek to calculate the electric potential on the surface of the head. In the inverse EEG problem, we are given the potential on the surface of the head and we seek to reconstruct the primary neuronal current that gave rise to this potential. The forward problem has a unique solution up to an additive constant [5], while for the inverse problem one can recover nothing more than the scalar function of the Helmholtz representation of the current [6]. In fact, the qualitative result that it is not possible to identify the current within a conductor from data collected outside the conductor was stated by Helmholtz 160 years ago [7]. However, the ultimate quantitative result on this problem was reached recently [6].

An excellent review of the electromagnetic activity of the human brain can be found in [8]. A standard book on the field 
is given in [1]. As far as the inverse source problem for the full Maxwell equations is concerned we refer to $[4,9]$.

Albanese and Monk [10] demonstrated that it is in general impossible to find even the support of a localized current in the interior of a conductor if this support has the same dimensionality with the conductor. This result was demonstrated in practice in [11] where it was assumed that the current is supported inside of a small sphere which is located in the interior of a larger conducting sphere. It was shown in [11] that it is impossible to recover the radius of the small sphere supporting the current. The Albanese and Monk result though is not true if the current lives in a set having a lower dimension and it is the purpose of the present work to demonstrate this result in practice.

In this work we solve the forward problem of EEG in the case where the current is continuously distributed along a small line segment, and then we try to solve the inverse problem of identifying the location, the orientation, the size, and the average dipolar moment. The inverse problem is reduced to a nonlinear algebraic system which can be handled by computational methods. However, we demonstrate an appropriate analytic manipulation of the system that reduces drastically the numerical interference, leading finally to a hybrid algorithm for the solution. With the help of this algorithm we provide a series of different solutions of the EEG inverse problem. Furthermore, two special cases are considered where the solution of the system is solved analytically.

The paper is organized as follows. Section 2 involves the mathematical formulation of the EEG problem and provides the solution of the forward problem in the fundamental case where the current is a single dipole. Then, in Section 3, we solve the forward problem when the current is distributed along a small line segment, and in Section 4 we develop the inverse problem that controls identification of this current. Finally, a thorough investigation of the inversion algorithm, developed in Section 4, is given in Section 5.

\section{The EEG Problem and Its Fundamental Solution}

We consider a sphere of radius $a$ to be a homogeneous conductor with conductivity $\sigma$ as the geometrical model of the total brain tissue. At the point $\mathbf{r}_{0}$, inside this sphere, a dipolar current with moment $\mathbf{Q}$ is activated, representing a synchronous excitation of a few thousand neurons. The generated current is then written as

$$
\mathbf{J}^{p}(\mathbf{r})=\mathbf{Q} \delta\left(\mathbf{r}-\mathbf{r}_{0}\right)
$$

while, according to the Quasi-Static Theory of Electromagnetism $[2,3]$, the generated electric potential $u^{-}$in the interior of the sphere has to solve the following Neumann boundary value problem:

$$
\begin{gathered}
\sigma \Delta_{\mathbf{r}} u^{-}\left(\mathbf{r} ; \mathbf{r}_{0}\right)=\mathbf{Q} \cdot \nabla_{\mathbf{r}} \delta\left(\mathbf{r}-\mathbf{r}_{0}\right), \quad r<a, \\
\frac{\partial}{\partial n} u^{-}\left(\mathbf{r} ; \mathbf{r}_{0}\right)=0, \quad r=a,
\end{gathered}
$$

where the operator $\partial / \partial n$ denotes the outward normal derivative on the surface of sphere.
Once the solution $u^{-}$is obtained, the electric potential $u^{+}$in the domain exterior to sphere satisfies the Dirichlet boundary value problem:

$$
\begin{gathered}
\sigma \Delta_{\mathbf{r}} u^{+}\left(\mathbf{r} ; \mathbf{r}_{0}\right)=0, \quad r>a, \\
u^{+}\left(\mathbf{r} ; \mathbf{r}_{0}\right)=u^{-}\left(\mathbf{r} ; \mathbf{r}_{0}\right), \quad r=a, \\
u^{+}\left(\mathbf{r} ; \mathbf{r}_{0}\right)=O\left(\frac{1}{r^{2}}\right), \quad r \longrightarrow \infty .
\end{gathered}
$$

The solution of Neumann problem (2), corresponding to zero value of the undetermined constant, is given in the form [5, 12]

$$
u^{-}\left(\mathbf{r} ; \mathbf{r}_{0}\right)=\frac{1}{4 \pi \sigma} \mathbf{Q} \cdot \frac{\mathbf{r}-\mathbf{r}_{0}}{\left|\mathbf{r}-\mathbf{r}_{0}\right|^{3}}+w(\mathbf{r})
$$

with

$$
\begin{aligned}
w(\mathbf{r})= & \frac{1}{\sigma}\left(\mathbf{Q} \cdot \nabla_{\mathbf{r}_{0}}\right) \\
& \times \sum_{n=1}^{\infty} \sum_{m=-n}^{n} \frac{n+1}{n(2 n+1)} \frac{r_{0}^{n} r^{n}}{a^{2 n+1}} Y_{n}^{m}\left(\widehat{\mathbf{r}}_{0}\right)^{*} Y_{n}^{m}(\widehat{\mathbf{r}}),
\end{aligned}
$$

where $Y_{n}^{m}$ stands for the normalized complex spherical harmonics

$$
Y_{n}^{m}(\widehat{\mathbf{r}})=\sqrt{\frac{2 n+1}{4 \pi} \frac{(n-|m|) !}{(n+|m|) !}} P_{n}^{|m|}(\cos \vartheta) \mathrm{e}^{i m \varphi}
$$

and $P_{n}^{m}$ denotes the Legendre functions of the first kind. The symbol “ " on the top of a vector denotes that the vector has unit length. In view of the addition theorem

$$
P_{n}\left(\widehat{\mathbf{r}} \cdot \widehat{\mathbf{r}}^{\prime}\right)=\frac{4 \pi}{2 n+1} \sum_{m=-n}^{n} Y_{n}^{m}(\widehat{\mathbf{r}}) Y_{n}^{m}\left(\widehat{\mathbf{r}}^{\prime}\right)^{*},
$$

where $P_{n}$ is the Legendre polynomial of degree $n$, the interior solution is written as

$$
\begin{aligned}
u^{-}\left(\mathbf{r} ; \mathbf{r}_{0}\right)= & \frac{1}{4 \pi \sigma} \mathbf{Q} \cdot \frac{\mathbf{r}-\mathbf{r}_{0}}{\left|\mathbf{r}-\mathbf{r}_{0}\right|^{3}}+\frac{1}{4 \pi \sigma}\left(\mathbf{Q} \cdot \nabla_{\mathbf{r}_{0}}\right) \\
& \times \sum_{n=1}^{\infty}\left(1+\frac{1}{n}\right) \frac{r_{0}^{n} r^{n}}{\alpha^{2 n+1}} P_{n}\left(\widehat{\mathbf{r}} \cdot \widehat{\mathbf{r}}_{0}\right) .
\end{aligned}
$$

Utilizing the Laplace expansion $[13,14]$

$$
\begin{aligned}
\frac{1}{\left|\mathbf{r}-\mathbf{r}^{\prime}\right|} & =\sum_{n=0}^{\infty} \sum_{m=-n}^{n} \frac{4 \pi}{2 n+1} \frac{r^{\prime n}}{r^{n+1}} Y_{n}^{m}(\widehat{\mathbf{r}}) Y_{n}^{m}\left(\widehat{\mathbf{r}}^{\prime}\right)^{*} \\
& =\sum_{n=0}^{\infty} \frac{r^{\prime n}}{r^{n+1}} P_{n}\left(\widehat{\mathbf{r}} \cdot \widehat{\mathbf{r}}^{\prime}\right)
\end{aligned}
$$

and evaluating the electric potential on the spherical boundary we arrive at the expression

$$
u^{-}\left(a \widehat{\mathbf{r}} ; \mathbf{r}_{0}\right)=\frac{1}{4 \pi \sigma}\left(\mathbf{Q} \cdot \nabla_{\mathbf{r}_{0}}\right) \sum_{n=1}^{\infty} \frac{2 n+1}{n} \frac{r_{0}^{n}}{\alpha^{n+1}} P_{n}\left(\widehat{\mathbf{r}} \cdot \widehat{\mathbf{r}}_{0}\right),
$$


where $a=|\mathbf{r}|$ and $\widehat{\mathbf{r}}$ is the unit vector in the direction of the observation point $\mathbf{r}$.

Then, using the boundary values (10) as the Dirichlet data for the exterior problem (3) we obtain the exterior potential [5]

$$
u^{+}\left(\mathbf{r} ; \mathbf{r}_{0}\right)=\frac{1}{4 \pi \sigma}\left(\mathbf{Q} \cdot \nabla_{\mathbf{r}_{0}}\right) \sum_{n=1}^{\infty} \frac{2 n+1}{n} \frac{r_{0}^{n}}{r^{n+1}} P_{n}\left(\widehat{\mathbf{r}} \cdot \widehat{\mathbf{r}}_{0}\right) .
$$

The solution $u^{-}\left(\mathbf{r} ; \mathbf{r}_{0}\right)$, given in (8), provides the solution of the interior problem (2), and the solution $u^{+}\left(\mathbf{r} ; \mathbf{r}_{0}\right)$, given in (11), provides the solution of the exterior problem (3), both for the case of a point excitation at $\mathbf{r}_{0}$. Therefore, they can be considered as the corresponding fundamental solutions for these two problems [15]. The relative solutions due to any distribution of current dipoles can be obtained by integrating these fundamental solutions over the source variable $\mathbf{r}_{0}$ [11].

\section{The Potential of a Linearly Distributed Current}

We assume that the neuronal current $\mathbf{J}^{p}$ is supported on a small segment of a smooth curve parametrically centered at the point $\mathbf{r}_{0}$. Let this curve be represented by the equation

$$
\mathbf{r}=\mathbf{r}(t), \quad t \in[-L, L], \quad \mathbf{r}(0)=\mathbf{r}_{0} .
$$

The neuronal current is then described by the function $\mathbf{J}^{p}(\mathbf{r}(t)), t \in[-L, L]$. Since the support curve has been assumed to be small we can approximate the current $\mathbf{J}^{p}(\mathbf{r}(t))$ by the linear part of its Taylor expansion; that is,

$$
\mathbf{J}^{p}(\mathbf{r}(t))=\mathbf{J}^{p}\left(\mathbf{r}_{0}\right)+t \frac{d \mathbf{r}(0)}{d t} \cdot\left[\nabla \otimes \mathbf{J}^{p}\left(\mathbf{r}_{0}\right)\right]+O\left(t^{2}\right) .
$$

In particular, if the curve is a small line segment of length $2 L$, centered at $\mathbf{r}_{0}$ and oriented along the direction $\widehat{\boldsymbol{\alpha}}=$ $\left(\alpha_{1}, \alpha_{2}, \alpha_{3}\right)$, that is,

$$
\mathbf{r}(t)=\mathbf{r}_{0}+t \widehat{\boldsymbol{\alpha}}, \quad t \in[-L, L],
$$

then representation (13) is written as

$$
\mathbf{J}^{p}(\mathbf{r}(t)) \approx \mathbf{Q}+t \mathbf{l}
$$

where $\mathbf{Q}=\left(Q_{1}, Q_{2}, Q_{3}\right)=\mathbf{J}^{p}\left(\mathbf{r}_{0}\right)$ provides an average moment and $\mathbf{l}=\left(l_{1}, l_{2}, l_{3}\right)=\widehat{\boldsymbol{\alpha}} \cdot \nabla \otimes \mathbf{J}^{p}\left(\mathbf{r}_{0}\right)$ provides an average directional derivative of the current along the direction $\widehat{\boldsymbol{\alpha}}$.

Next we calculate the total potential which is generated by the approximate current (15). In fact, since our ultimate goal is to invert the EEG data that will give us the quantities $\mathbf{Q}, \mathbf{r}_{0}$, $\widehat{\boldsymbol{\alpha}}$, and $L$ we will calculate as many terms of expansion (11) as we actually need.

Formula (11), for the excitation dipole $\left\{\mathbf{r}^{\prime}, \mathbf{J}\left(\mathbf{r}^{\prime}\right)\right\}$, is written as

$$
\begin{gathered}
u^{+}\left(\mathbf{r} ; \mathbf{r}^{\prime}\right) \\
=\frac{\mathbf{J}^{p}\left(\mathbf{r}^{\prime}\right)}{4 \pi \sigma} \cdot\left[\frac{3}{r^{2}} \nabla_{\mathbf{r}^{\prime}}\left(r^{\prime} P_{1}\left(\widehat{\mathbf{r}} \cdot \widehat{\mathbf{r}}^{\prime}\right)\right)+\frac{5}{2 r^{3}} \nabla_{\mathbf{r}^{\prime}}\left(r^{\prime 2} P_{2}\left(\widehat{\mathbf{r}} \cdot \widehat{\mathbf{r}}^{\prime}\right)\right)\right. \\
\left.+\frac{7}{3 r^{4}} \nabla_{\mathbf{r}^{\prime}}\left(r^{\prime 3} P_{3}\left(\widehat{\mathbf{r}} \cdot \widehat{\mathbf{r}}^{\prime}\right)\right)\right]+O\left(r^{-5}\right) .
\end{gathered}
$$

Using the standard expressions of the Legendre polynomials [14] and performing the indicated calculation, we obtain the following relations, which are written in dyadic form [16] in order to isolate the factors that are going to be integrated:

$$
\begin{aligned}
& \nabla_{\mathbf{r}^{\prime}}\left(r^{\prime} P_{1}\left(\widehat{\mathbf{r}} \cdot \widehat{\mathbf{r}}^{\prime}\right)\right)= \widehat{\mathbf{r}}, \\
& \nabla_{\mathbf{r}^{\prime}}\left(r^{\prime 2} P_{2}\left(\widehat{\mathbf{r}} \cdot \widehat{\mathbf{r}}^{\prime}\right)\right)=(3 \widehat{\mathbf{r}} \otimes \widehat{\mathbf{r}}-\widetilde{\mathbf{I}}) \cdot \mathbf{r}^{\prime} \\
&=(3 \widehat{\mathbf{r}} \otimes \widehat{\mathbf{r}}-\widetilde{\mathbf{I}}) \cdot\left(\mathbf{r}_{0}+t \widehat{\boldsymbol{\alpha}}\right), \\
& \nabla_{\mathbf{r}^{\prime}}\left(r^{\prime 3} P_{3}\left(\widehat{\mathbf{r}} \cdot \widehat{\mathbf{r}}^{\prime}\right)\right)= \frac{3}{2}(5 \widehat{\mathbf{r}} \otimes \widehat{\mathbf{r}} \otimes \widehat{\mathbf{r}}-2 \widetilde{\mathbf{I}} \otimes \widehat{\mathbf{r}}-\widehat{\mathbf{r}} \otimes \widetilde{\mathbf{I}}): \mathbf{r}^{\prime} \otimes \mathbf{r}^{\prime} \\
&= \frac{3}{2}(5 \widehat{\mathbf{r}} \otimes \widehat{\mathbf{r}} \otimes \widehat{\mathbf{r}}-2 \widetilde{\mathbf{I}} \\
&\qquad \widehat{\mathbf{r}}-\widehat{\mathbf{r}} \otimes \widetilde{\mathbf{I}}):\left(\mathbf{r}_{0}+t \widehat{\boldsymbol{\alpha}}\right) \otimes\left(\mathbf{r}_{0}+t \widehat{\boldsymbol{\alpha}}\right) .
\end{aligned}
$$

The symbol $\widetilde{I}$ denotes the identity dyadic, and ":" defines the double contraction [16]:

$$
(\mathbf{a} \otimes \mathbf{b}):(\mathbf{c} \otimes \mathbf{d})=(\mathbf{b} \cdot \mathbf{c})(\mathbf{a} \cdot \mathbf{d})
$$

and similarly the triple contraction is defined as

$$
(\mathbf{a} \otimes \mathbf{b} \otimes \mathbf{c}) \vdots(\mathbf{d} \otimes \mathbf{e} \otimes \mathbf{f})=(\mathbf{c} \cdot \mathbf{d})(\mathbf{b} \cdot \mathbf{e})(\mathbf{a} \cdot \mathbf{f})
$$

On the other hand, the exterior potential given in (16) can be written in its Cartesian form $[11,12]$ as follows:

$$
u^{+}\left(\mathbf{r} ; \mathbf{r}^{\prime}\right)=\frac{1}{4 \pi \sigma}\left[\frac{H_{1}(\mathbf{r})}{r^{3}}+\frac{H_{2}(\mathbf{r})}{r^{5}}+\frac{H_{3}(\mathbf{r})}{r^{7}}\right]+O\left(r^{-5}\right),
$$

where the coefficients

$$
\begin{aligned}
& H_{1}(\mathbf{r})=3 \mathbf{r} \cdot \mathbf{J}^{p}\left(\mathbf{r}^{\prime}\right) \\
& H_{2}(\mathbf{r})=\frac{5}{2}\left(3 \mathbf{r} \otimes \mathbf{r}-r^{2} \widetilde{\mathbf{I}}\right): \mathbf{r}^{\prime} \otimes \mathbf{J}^{p}\left(\mathbf{r}^{\prime}\right) \\
& H_{3}(\mathbf{r})=\frac{7}{2}\left(5 \mathbf{r} \otimes \mathbf{r} \otimes \mathbf{r}-2 r^{2} \widetilde{\mathbf{I}} \otimes \mathbf{r}-r^{2} \mathbf{r} \otimes \widetilde{\mathbf{I}}\right) \vdots \mathbf{r}^{\prime} \otimes \mathbf{r}^{\prime} \otimes \mathbf{J}^{p}\left(\mathbf{r}^{\prime}\right)
\end{aligned}
$$

are homogeneous harmonic functions [11].

All we need to do then is to insert expressions (14) and (15) in (21) and integrate the resulting equations with respect to $t$ 
from $-L$ to $L$. Performing these long calculations we arrive at the expressions

$$
\begin{aligned}
H_{1}(\mathbf{r})= & 3 \mathbf{r} \cdot \int_{-L}^{L}(\mathbf{Q}+t \mathbf{l}) d t \\
= & 6 L \mathbf{r} \cdot \mathbf{Q}, \\
H_{2}(\mathbf{r})= & \frac{5}{2}\left(3 \mathbf{r} \otimes \mathbf{r}-r^{2} \widetilde{\mathbf{I}}\right): \int_{-L}^{L}\left(\mathbf{r}_{0}+t \widehat{\boldsymbol{\alpha}}\right) \otimes(\mathbf{Q}+t \mathbf{l}) d t \\
= & \frac{5}{3}\left(3 \mathbf{r} \otimes \mathbf{r}-r^{2} \widetilde{\mathbf{I}}\right):\left(3 L \mathbf{r}_{0} \otimes \mathbf{Q}+L^{3} \widehat{\boldsymbol{\alpha}} \otimes \mathbf{l}\right), \\
H_{3}(\mathbf{r})= & \frac{7}{2}\left(5 \mathbf{r} \otimes \mathbf{r} \otimes \mathbf{r}-2 r^{2} \widetilde{\mathbf{I}} \otimes \mathbf{r}-r^{2} \mathbf{r} \otimes \widetilde{\mathbf{I}}\right) \\
\vdots & \int_{-L}^{L}\left(\mathbf{r}_{0}+t \widehat{\boldsymbol{\alpha}}\right) \otimes\left(\mathbf{r}_{0}+t \widehat{\boldsymbol{\alpha}}\right) \otimes(\mathbf{Q}+t \mathbf{l}) d t \\
= & \frac{7}{3}\left(5 \mathbf{r} \otimes \mathbf{r} \otimes \mathbf{r}-2 r^{2} \widetilde{\mathbf{I}} \otimes \mathbf{r}-r^{2} \mathbf{r} \otimes \widetilde{\mathbf{I}}\right) \\
\vdots & {\left[3 L \mathbf{r}_{0} \otimes \mathbf{r}_{0} \otimes \mathbf{Q}\right.} \\
& \left.+L^{3}\left(\widehat{\boldsymbol{\alpha}} \otimes \widehat{\boldsymbol{\alpha}} \otimes \mathbf{Q}+\mathbf{r}_{0} \otimes \widehat{\boldsymbol{\alpha}} \otimes \mathbf{l}+\widehat{\boldsymbol{\alpha}} \otimes \mathbf{r}_{0} \otimes \mathbf{l}\right)\right] .
\end{aligned}
$$

After we replace the above expressions of the harmonic functions $H_{1}, H_{2}, H_{3}$ in expansion (20) we arrive at the Cartesian representation of the exterior potential $u^{+}$up to the terms of order $r^{-5}$. That solves the relative forward EEG problem for a neuronal excitation that is supported on a small line segment.

\section{The Structure of the Inverse Problem}

Since the harmonic functions $H_{1}, H_{2}$, and $H_{3}$ are homogeneous polynomials of degrees 1,2 , and 3 , respectively, it follows that

$$
\begin{gathered}
H_{1}(\mathbf{r})=A_{1} x_{1}+A_{2} x_{2}+A_{3} x_{3}, \\
H_{2}(\mathbf{r})=B_{1} x_{1}^{2}+B_{2} x_{2}^{2}+B_{3} x_{3}^{2}+B_{12} x_{1} x_{2}+B_{23} x_{2} x_{3} \\
+B_{31} x_{3} x_{1}
\end{gathered}
$$

with

$$
\begin{gathered}
B_{1}+B_{2}+B_{3}=0 \\
H_{3}(\mathbf{r})=C_{1} x_{1}^{3}+C_{2} x_{2}^{3}+C_{3} x_{3}^{3} \\
+C_{12} x_{1}^{2} x_{2}+C_{21} x_{2}^{2} x_{1}+C_{23} x_{2}^{2} x_{3} \\
+C_{32} x_{3}^{2} x_{2}+C_{31} x_{3}^{2} x_{1}+C_{13} x_{1}^{2} x_{3}+C_{123} x_{1} x_{2} x_{3}
\end{gathered}
$$

with

$$
\begin{aligned}
& 3 C_{1}+C_{21}+C_{31}=0, \\
& C_{12}+3 C_{2}+C_{32}=0, \\
& C_{13}+C_{23}+3 C_{3}=0 .
\end{aligned}
$$

Assume the idealized case where the exterior potential $u^{+}$ is known. Then, expansion (20) is known and therefore the coefficients $A, B$, and $C$ are also known. Hence, if we rewrite the polynomials $H_{1}, H_{2}$, and $H_{3}$ in terms of the Cartesian monomials that appear in (25), (26), and (28), then we can utilize their linear independence to equate each monomial with the corresponding known coefficient $A, B$, or $C$.

Actually, the EEG data are given as potential differences on the boundary of the head, but this is equivalent to knowing the exterior field for the following reason. Let $f(\vartheta, \varphi)$ be the given data on the sphere of radius $\alpha$. Then, $f$ can be expanded in the spherical harmonics $Y_{n}^{m}$ and let $A_{n}^{m}$ be the coefficients of this expansion. The function $f$ is the trace of the exterior harmonic function $u^{+}$, which also has an expansion in the spherical harmonics $r^{-(n+1)} Y_{n}^{m}$ with coefficients $B_{n}^{m}$. It is obvious that $B_{n}^{m}=a^{n+1} A_{n}^{m}$. Consequently, the EEG data on the surface of the sphere uniquely specify the exterior harmonic field $u^{+}$.

Equations (22) and (25) imply immediately that

$$
\mathbf{Q}=\frac{1}{6 L}\left(A_{1}, A_{2}, A_{3}\right)=\frac{1}{6 L} \mathbf{A} .
$$

We remind that $x_{01}, x_{02}$, and $x_{03}$ are the three components of the vector $\mathbf{r}_{0}$, representing the middle point of the line segment, and $\alpha_{1}, \alpha_{2}, \alpha_{3}$ are the directional cosines of the direction $\widehat{\boldsymbol{\alpha}}$ of the line segment. Then, from (23), (26), and (30) we obtain the six relations

$$
\begin{aligned}
B_{1} & =\frac{5}{2} A_{1} x_{01}-\frac{5}{6}\left(\mathbf{A} \cdot \mathbf{r}_{0}\right)+5 L^{3} a_{1} l_{1}-\frac{5}{3} L^{3}(\widehat{\boldsymbol{\alpha}} \cdot \mathbf{l}), \\
B_{2} & =\frac{5}{2} A_{2} x_{02}-\frac{5}{6}\left(\mathbf{A} \cdot \mathbf{r}_{0}\right)+5 L^{3} a_{2} l_{2}-\frac{5}{3} L^{3}(\widehat{\boldsymbol{\alpha}} \cdot \mathbf{l}), \\
B_{3} & =\frac{5}{2} A_{3} x_{03}-\frac{5}{6}\left(\mathbf{A} \cdot \mathbf{r}_{0}\right)+5 L^{3} a_{3} l_{3}-\frac{5}{3} L^{3}(\widehat{\boldsymbol{\alpha}} \cdot \mathbf{l}), \\
B_{12} & =\frac{5}{2}\left(A_{1} x_{02}+A_{2} x_{01}\right)+5 L^{3}\left(\alpha_{1} l_{2}+\alpha_{2} l_{1}\right), \\
B_{23} & =\frac{5}{2}\left(A_{2} x_{03}+A_{3} x_{02}\right)+5 L^{3}\left(\alpha_{2} l_{3}+\alpha_{3} l_{2}\right), \\
B_{31} & =\frac{5}{2}\left(A_{1} x_{03}+A_{3} x_{01}\right)+5 L^{3}\left(\alpha_{1} l_{3}+\alpha_{3} l_{1}\right)
\end{aligned}
$$

and constraint (27) is obviously satisfied.

Finally, (24), (28), and (30) provide three relations from the cubic terms $x_{i}^{3}$

$$
\begin{aligned}
C_{1}= & \frac{7}{6}\left(5 x_{01}^{2}-r_{0}^{2}\right) A_{1}-\frac{7}{3} x_{01}\left(\mathbf{A} \cdot \mathbf{r}_{0}\right) \\
& +\frac{7}{18} L^{2}\left(5 \alpha_{1}^{2}-1\right) A_{1}-\frac{7}{9} L^{2} \alpha_{1}(\mathbf{A} \cdot \widehat{\boldsymbol{\alpha}}) \\
& +\frac{14}{3} L^{3}\left[3 x_{01} \alpha_{1} l_{1}-x_{01}(\widehat{\boldsymbol{\alpha}} \cdot \mathbf{l})-x_{02}\left(\alpha_{1} l_{2}+\alpha_{2} l_{1}\right)\right. \\
& \left.-x_{03}\left(\alpha_{1} l_{3}+\alpha_{3} l_{1}\right)\right],
\end{aligned}
$$




$$
\begin{aligned}
C_{2}= & \frac{7}{6}\left(5 x_{02}^{2}-r_{0}^{2}\right) A_{2}-\frac{7}{3} x_{02}\left(\mathbf{A} \cdot \mathbf{r}_{0}\right) \\
& +\frac{7}{18} L^{2}\left(5 \alpha_{2}^{2}-1\right) A_{2}-\frac{7}{9} L^{2} \alpha_{2}(\mathbf{A} \cdot \widehat{\boldsymbol{\alpha}}) \\
& +\frac{14}{3} L^{3}\left[3 x_{02} \alpha_{2} l_{2}-x_{02}(\widehat{\boldsymbol{\alpha}} \cdot \mathbf{l})\right. \\
C_{3}= & \frac{7}{6}\left(5 x_{03}^{2}-r_{0}^{2}\right) A_{3}-\frac{7}{3} x_{03}\left(\mathbf{A} \cdot \mathbf{r}_{0}\right) \\
& +\frac{7}{18} L^{2}\left(5 \alpha_{3}^{2}-1\right) A_{3}-\frac{7}{9} L^{2} \alpha_{3}(\mathbf{A} \cdot \widehat{\boldsymbol{\alpha}}) \\
& +\frac{14}{3} L^{3}\left[3 x_{03} \alpha_{3} l_{3}-x_{03}(\widehat{\boldsymbol{\alpha}} \cdot \mathbf{l})\right. \\
& \left.\quad-x_{01}\left(\alpha_{1} l_{3}+\alpha_{3} l_{1}\right)-x_{02}\left(\alpha_{2} l_{3}+\alpha_{3} l_{2}\right)\right]
\end{aligned}
$$

six relations from the cross terms $x_{i}^{2} x_{j}, i \neq j$,

$$
\begin{aligned}
& C_{12}=\frac{35}{6}\left(x_{01}^{2} A_{2}+2 x_{01} x_{02} A_{1}\right)+\frac{35}{18} L^{2}\left(\alpha_{1}^{2} A_{2}+2 \alpha_{1} \alpha_{2} A_{1}\right) \\
& +\frac{70}{3} L^{3}\left(x_{01} \alpha_{1} l_{2}+x_{01} \alpha_{2} l_{1}+x_{02} \alpha_{1} l_{1}\right) \\
& -\frac{7}{3} x_{02}\left(\mathbf{A} \cdot \mathbf{r}_{0}\right)-\frac{7}{9} L^{2} \alpha_{2}(\mathbf{A} \cdot \widehat{\boldsymbol{\alpha}})-\frac{14}{3} L^{3} x_{02}(\widehat{\boldsymbol{\alpha}} \cdot \mathbf{l}) \\
& -\frac{14}{3} L^{3} \alpha_{2}\left(\mathbf{r}_{0} \cdot \mathbf{l}\right)-\frac{7}{6} r_{0}^{2} A_{2}-\frac{7}{18} L^{2} A_{2} \\
& -\frac{14}{3} L^{3} l_{2}\left(\widehat{\boldsymbol{\alpha}} \cdot \mathbf{r}_{0}\right) \text {, } \\
& C_{21}=\frac{35}{6}\left(x_{02}^{2} A_{1}+2 x_{01} x_{02} A_{2}\right)+\frac{35}{18} L^{2}\left(\alpha_{2}^{2} A_{1}+2 \alpha_{1} \alpha_{2} A_{2}\right) \\
& +\frac{70}{3} L^{3}\left(x_{02} \alpha_{2} l_{1}+x_{02} \alpha_{1} l_{2}+x_{01} \alpha_{2} l_{2}\right) \\
& -\frac{7}{3} x_{01}\left(\mathbf{A} \cdot \mathbf{r}_{0}\right)-\frac{7}{9} L^{2} \alpha_{1}(\mathbf{A} \cdot \widehat{\boldsymbol{\alpha}})-\frac{14}{3} L^{3} x_{01}(\widehat{\boldsymbol{\alpha}} \cdot \mathbf{l}) \\
& -\frac{14}{3} L^{3} \alpha_{1}\left(\mathbf{r}_{0} \cdot \mathbf{l}\right)-\frac{7}{6} r_{0}^{2} A_{1}-\frac{7}{18} L^{2} A_{1} \\
& -\frac{14}{3} L^{3} l_{1}\left(\widehat{\boldsymbol{\alpha}} \cdot \mathbf{r}_{0}\right) \\
& C_{23}=\frac{35}{6}\left(x_{02}^{2} A_{3}+2 x_{02} x_{03} A_{2}\right)+\frac{35}{18} L^{2}\left(\alpha_{2}^{2} A_{3}+2 \alpha_{2} \alpha_{3} A_{2}\right) \\
& +\frac{70}{3} L^{3}\left(x_{02} \alpha_{2} l_{3}+x_{02} \alpha_{3} l_{2}+x_{03} \alpha_{2} l_{2}\right) \\
& -\frac{7}{3} x_{03}\left(\mathbf{A} \cdot \mathbf{r}_{0}\right)-\frac{7}{9} L^{2} \alpha_{3}(\mathbf{A} \cdot \widehat{\boldsymbol{\alpha}})-\frac{14}{3} L^{3} x_{03}(\widehat{\boldsymbol{\alpha}} \cdot \mathbf{l}) \\
& -\frac{14}{3} L^{3} \alpha_{3}\left(\mathbf{r}_{0} \cdot \mathbf{l}\right)-\frac{7}{6} r_{0}^{2} A_{3}-\frac{7}{18} L^{2} A_{3} \\
& -\frac{14}{3} L^{3} l_{3}\left(\widehat{\boldsymbol{\alpha}} \cdot \mathbf{r}_{0}\right)
\end{aligned}
$$

$$
\begin{aligned}
C_{32}= & \frac{35}{6}\left(x_{03}^{2} A_{2}+2 x_{02} x_{03} A_{3}\right)+\frac{35}{18} L^{2}\left(\alpha_{3}^{2} A_{2}+2 \alpha_{2} \alpha_{3} A_{3}\right) \\
& +\frac{70}{3} L^{3}\left(x_{03} \alpha_{3} l_{2}+x_{03} \alpha_{2} l_{3}+x_{02} \alpha_{3} l_{3}\right) \\
& -\frac{7}{3} x_{02}\left(\mathbf{A} \cdot \mathbf{r}_{0}\right)-\frac{7}{9} L^{2} \alpha_{2}(\mathbf{A} \cdot \widehat{\boldsymbol{\alpha}})-\frac{14}{3} L^{3} x_{02}(\widehat{\boldsymbol{\alpha}} \cdot \mathbf{l}) \\
& -\frac{14}{3} L^{3} \alpha_{2}\left(\mathbf{r}_{0} \cdot \mathbf{l}\right)-\frac{7}{6} r_{0}^{2} A_{2}-\frac{7}{18} L^{2} A \\
& -\frac{14}{3} L^{3} l_{2}\left(\widehat{\boldsymbol{\alpha}} \cdot \mathbf{r}_{0}\right), \\
C_{31}= & \frac{35}{6}\left(x_{03}^{2} A_{1}+2 x_{01} x_{03} A_{3}\right)+\frac{35}{18} L^{2}\left(\alpha_{3}^{2} A_{1}+2 \alpha_{1} \alpha_{3} A_{3}\right) \\
& +\frac{70}{3} L^{3}\left(x_{03} \alpha_{3} l_{1}+x_{03} \alpha_{1} l_{3}+x_{01} \alpha_{3} l_{3}\right) \\
& -\frac{7}{3} x_{01}\left(\mathbf{A} \cdot \mathbf{r}_{0}\right)-\frac{7}{9} L^{2} \alpha_{1}(\mathbf{A} \cdot \widehat{\boldsymbol{\alpha}})-\frac{14}{3} L^{3} x_{01}(\widehat{\boldsymbol{\alpha}} \cdot \mathbf{l}) \\
& \left.-\frac{14}{3} L^{3} \alpha_{3}\left(\mathbf{r}_{0} \cdot \mathbf{l}\right)-\frac{7}{6} r_{0}^{2} A_{3}-\frac{7}{18} L^{2} A_{3} \cdot \mathbf{r}_{0}\right), \\
& -\frac{14}{3} L^{3} \alpha_{1}\left(\mathbf{r}_{0} \cdot \mathbf{l}\right)-\frac{7}{6} r_{0}^{2} A_{1}-\frac{7}{18} L^{2} A_{1} \\
C_{13}= & \frac{35}{6}\left(x_{01}^{2} A_{3}+2 x_{01} x_{03} A_{1}\right)+\frac{35}{18} L^{2}\left(x_{01}^{2} \alpha_{1} l_{3}+2 \alpha_{1} \alpha_{3} A_{1}\right) \\
& -\frac{14}{3} L^{3} l_{1}\left(\widehat{\boldsymbol{\alpha}} \cdot \mathbf{r}_{01} \alpha_{3} l_{1}+x_{03} \alpha_{1} l_{1}\right) \\
& \\
& \\
&
\end{aligned}
$$

and one relation from the product term $x_{1} x_{2} x_{3}$

$$
\begin{aligned}
C_{123}= & \frac{35}{3}\left(x_{01} x_{02} A_{3}+x_{02} x_{03} A_{1}+x_{03} x_{01} A_{2}\right) \\
& +\frac{35}{9} L^{2}\left(\alpha_{1} \alpha_{2} A_{3}+\alpha_{2} \alpha_{3} A_{1}+\alpha_{1} \alpha_{3} A_{2}\right) \\
& +\frac{70}{3} L^{3}\left(x_{01} \alpha_{2} l_{3}+x_{02} \alpha_{3} l_{1}+x_{03} \alpha_{1} l_{2}\right. \\
& \left.+x_{01} \alpha_{3} l_{2}+x_{03} \alpha_{2} l_{1}+x_{02} \alpha_{1} l_{3}\right),
\end{aligned}
$$

where it is easy to verify that the three constraints (29) hold true.

Equations (30)-(34) define a nonlinear system of 19 equations, 15 of which are linearly independent because of constraints (27) and (29), for the determination of the 12 independent unknowns $\mathbf{Q}, \mathbf{r}_{0}, \widehat{\boldsymbol{\alpha}}, \mathbf{l}$, and $L$, since only two out 
of the three components of $\widehat{\boldsymbol{\alpha}}$ are independent. In fact, we can use relations (31) to reduce the above system to

$$
\begin{aligned}
C_{1}= & \frac{14}{15}\left[3 B_{1} x_{01}-B_{12} x_{02}-B_{31} x_{03}\right] \\
& +\frac{7}{6}\left[\left(r_{0}^{2}-5 x_{01}^{2}\right) A_{1}+2 x_{01}\left(\mathbf{A} \cdot \mathbf{r}_{0}\right)\right] \\
& +\frac{7}{18} L^{2}\left[\left(5 \alpha_{1}^{2}-1\right) A_{1}-2 \alpha_{1}(\mathbf{A} \cdot \widehat{\boldsymbol{\alpha}})\right] \\
C_{2}= & \frac{14}{15}\left[3 B_{2} x_{02}-B_{12} x_{01}-B_{23} x_{03}\right] \\
& +\frac{7}{6}\left[\left(r_{0}^{2}-5 x_{02}^{2}\right) A_{2}+2 x_{02}\left(\mathbf{A} \cdot \mathbf{r}_{0}\right)\right] \\
& +\frac{7}{18} L^{2}\left[\left(5 \alpha_{2}^{2}-1\right) A_{2}-2 \alpha_{2}(\mathbf{A} \cdot \widehat{\boldsymbol{\alpha}})\right] \\
C_{3}= & \frac{14}{15}\left[3 B_{3} x_{03}-B_{23} x_{02}-B_{31} x_{01}\right] \\
& +\frac{7}{6}\left[\left(r_{0}^{2}-5 x_{03}^{2}\right) A_{3}+2 x_{03}\left(\mathbf{A} \cdot \mathbf{r}_{0}\right)\right] \\
& +\frac{7}{18} L^{2}\left[\left(5 \alpha_{3}^{2}-1\right) A_{3}-2 \alpha_{3}(\mathbf{A} \cdot \widehat{\boldsymbol{\alpha}})\right] \\
& +\frac{7}{18} L^{2}\left[\left(5 \alpha_{1}^{2}-1\right) A_{2}-2 \alpha_{2}(\mathbf{A} \cdot \widehat{\boldsymbol{\alpha}})+10 A_{1} \alpha_{1} \alpha_{2}\right] \\
C_{12}= & \left.\frac{7}{6}\left[r_{0}^{2}-5 x_{01}^{2}\right) A_{2}+2 x_{02}\left(\mathbf{A} \cdot \mathbf{r}_{0}\right)-10 A_{1} x_{01} x_{02}\right] \\
& +\frac{14}{15}\left[4 B_{12} x_{01}+\left(5 B_{1}-2 B_{2}\right) x_{02}-B_{23} x_{03}\right] \\
& \\
&
\end{aligned}
$$

$$
\begin{aligned}
C_{21}= & \frac{7}{6}\left[\left(r_{0}^{2}-5 x_{02}^{2}\right) A_{1}+2 x_{01}\left(\mathbf{A} \cdot \mathbf{r}_{0}\right)-10 A_{2} x_{01} x_{02}\right] \\
& +\frac{14}{15}\left[\left(5 B_{2}-2 B_{1}\right) x_{01}+4 B_{12} x_{02}-B_{31} x_{03}\right] \\
& +\frac{7}{18} L^{2}\left[\left(5 \alpha_{2}^{2}-1\right) A_{1}-2 \alpha_{1}(\mathbf{A} \cdot \widehat{\boldsymbol{\alpha}})+10 A_{2} \alpha_{1} \alpha_{2}\right]
\end{aligned}
$$

$$
\begin{aligned}
C_{23}= & \frac{7}{6}\left[\left(r_{0}^{2}-5 x_{02}^{2}\right) A_{3}+2 x_{03}\left(\mathbf{A} \cdot \mathbf{r}_{0}\right)-10 A_{2} x_{02} x_{03}\right] \\
& +\frac{14}{15}\left[-B_{31} x_{01}+4 B_{23} x_{02}+\left(5 B_{2}-2 B_{3}\right) x_{03}\right] \\
& +\frac{7}{18} L^{2}\left[\left(5 \alpha_{2}^{2}-1\right) A_{3}-2 \alpha_{3}(\mathbf{A} \cdot \widehat{\boldsymbol{\alpha}})+10 A_{2} \alpha_{2} \alpha_{3}\right]
\end{aligned}
$$

$$
\begin{aligned}
C_{32}= & \frac{7}{6}\left[\left(r_{0}^{2}-5 x_{03}^{2}\right) A_{2}+2 x_{02}\left(\mathbf{A} \cdot \mathbf{r}_{0}\right)-10 A_{3} x_{02} x_{03}\right] \\
& +\frac{14}{15}\left[-B_{12} x_{01}+\left(5 B_{3}-2 B_{2}\right) x_{02}+4 B_{23} x_{03}\right] \\
& +\frac{7}{18} L^{2}\left[\left(5 \alpha_{3}^{2}-1\right) A_{2}-2 \alpha_{2}(\mathbf{A} \cdot \widehat{\boldsymbol{\alpha}})+10 A_{3} \alpha_{2} \alpha_{3}\right]
\end{aligned}
$$

$$
\begin{aligned}
C_{31}= & \frac{7}{6}\left[\left(r_{0}^{2}-5 x_{03}^{2}\right) A_{1}+2 x_{01}\left(\mathbf{A} \cdot \mathbf{r}_{0}\right)-10 A_{3} x_{01} x_{03}\right] \\
& +\frac{14}{15}\left[\left(5 B_{3}-2 B_{1}\right) x_{01}-B_{12} x_{02}+4 B_{31} x_{03}\right] \\
& +\frac{7}{18} L^{2}\left[\left(5 \alpha_{3}^{2}-1\right) A_{1}-2 \alpha_{1}(\mathbf{A} \cdot \widehat{\boldsymbol{\alpha}})+10 A_{3} \alpha_{1} \alpha_{3}\right] \\
C_{13}= & \frac{7}{6}\left[\left(r_{0}^{2}-5 x_{01}^{2}\right) A_{3}+2 x_{03}\left(\mathbf{A} \cdot \mathbf{r}_{0}\right)-10 A_{1} x_{01} x_{03}\right] \\
& +\frac{14}{15}\left[4 B_{31} x_{01}-B_{23} x_{02}+\left(5 B_{1}-2 B_{3}\right) x_{03}\right] \\
& +\frac{7}{18} L^{2}\left[\left(5 \alpha_{1}^{2}-1\right) A_{3}-2 \alpha_{3}(\mathbf{A} \cdot \widehat{\boldsymbol{\alpha}})+10 A_{1} \alpha_{1} \alpha_{3}\right] \\
& +\frac{35}{9} L^{2}\left(A_{1} \alpha_{2} \alpha_{3}+A_{2} \alpha_{1} \alpha_{3}+A_{3} \alpha_{1} \alpha_{2}\right) \\
C_{123}= & -\frac{35}{3}\left(A_{1} x_{02} x_{03}+A_{2} x_{01} x_{03}+A_{3} x_{01} x_{02}\right) \\
& +\frac{14}{3}\left(B_{23} x_{01}+B_{31} x_{02}+B_{12} x_{03}\right) \\
&
\end{aligned}
$$

The system of 7 independent equations (35)-(44) (10 equations minus 3 constraints) involves only the 6 unknowns $\mathbf{r}_{0}, \widehat{\boldsymbol{\alpha}}$, and $L$, since again only two components of $\widehat{\boldsymbol{\alpha}}$ are independent. Therefore, the solution of this system provides the position of the middle point $\mathbf{r}_{0}$ of the line segment, the orientation $\widehat{\boldsymbol{\alpha}}$ of the line segment, and its length $2 L$. Finally, inserting the length $L$ in (30) we obtain the average moment $\mathbf{Q}$. This way we obtain a good approximation of the linearly supported neuronal current and therefore the solution of the inverse EEG problem.

Obviously, it is extremely difficult, if possible, to solve the above nonlinear system without the use of a computational machine, but it is straightforward to obtain the numerical solution once the $A, B$, and $C$ constants are inserted into the system. Numerical tests show that there is exactly one real solution of this system modulo the orientation of the line segment. However, a further analytical manipulation of the system is possible, as demonstrated in the next section.

\section{A Hybrid Investigation of the Inversion Algorithm}

First, we utilize the identity

$$
\alpha_{1}^{2}+\alpha_{2}^{2}+\alpha_{3}^{2}=1
$$

and introduce the scaling

$$
L \alpha_{i}=a_{i}, \quad i=1,2,3
$$

in order for the system to contain only the six unknowns $x_{01}$, $x_{02}$, and $x_{03}$ and $a_{1}, a_{2}$, and $a_{3}$. Next, we can ignore (40), (41), 
and (42) since they are automatically satisfied because of (27) and (29). Then the reduced system reads

$$
\begin{aligned}
C_{1}=\frac{14}{15}\left(3 B_{1} x_{01}-B_{12} x_{02}-B_{31} x_{03}\right) \\
-\frac{7}{6}\left[A_{1}\left(4 x_{01}^{2}-x_{02}^{2}-x_{03}^{2}\right)\right. \\
\left.\quad-2 x_{01}\left(A_{1} x_{01}+A_{2} x_{02}+A_{3} x_{03}\right)\right] \\
+\frac{7}{18}\left[A_{1}\left(4 a_{1}^{2}-a_{2}^{2}-a_{3}^{2}\right)\right. \\
\left.\quad-2 a_{1}\left(a_{1} A_{1}+a_{2} A_{2}+a_{3} A_{3}\right)\right] \\
C_{2}=\frac{14}{15}\left(-B_{12} x_{01}+3 B_{2} x_{02}-B_{23} x_{03}\right) \\
-\frac{7}{6}\left[A_{2}\left(-x_{01}^{2}+4 x_{02}^{2}-x_{03}^{2}\right)\right. \\
\left.\quad-2 x_{02}\left(A_{1} x_{01}+A_{2} x_{02}+A_{3} x_{03}\right)\right] \\
+\frac{7}{18}\left[A_{2}\left(-a_{1}^{2}+4 a_{2}^{2}-a_{3}^{2}\right)\right. \\
\left.\quad-2 a_{2}\left(a_{1} A_{1}+a_{2} A_{2}+a_{3} A_{3}\right)\right]
\end{aligned}
$$$$
C_{3}=\frac{14}{15}\left(-B_{23} x_{02}+3 B_{3} x_{03}-B_{31} x_{01}\right)
$$$$
-\frac{7}{6}\left[A_{3}\left(-x_{01}^{2}-x_{02}^{2}+4 x_{03}^{2}\right)\right.
$$$$
\left.-2 x_{03}\left(A_{1} x_{01}+A_{2} x_{02}+A_{3} x_{03}\right)\right]
$$$$
+\frac{7}{18}\left[A_{3}\left(-a_{1}^{2}-a_{2}^{2}+4 a_{3}^{2}\right)\right.
$$$$
\left.-2 a_{3}\left(a_{1} A_{1}+a_{2} A_{2}+a_{3} A_{3}\right)\right],
$$$$
C_{12}=\frac{7}{6}\left[2 x_{02}\left(A_{1} x_{01}+A_{2} x_{02}+A_{3} x_{03}\right)-10 A_{1} x_{01} x_{02}\right.
$$$$
\left.+A_{2}\left(-4 x_{01}^{2}+x_{02}^{2}+x_{03}^{2}\right)\right]
$$$$
+\frac{14}{15}\left[x_{02}\left(4 B_{1}-3 B_{2}-B_{3}\right)+4 B_{12} x_{01}-B_{23} x_{03}\right]
$$$$
+\frac{7}{18}\left[A_{2}\left(4 a_{1}^{2}-a_{2}^{2}-a_{3}^{2}\right)\right.
$$$$
\left.-2 a_{2}\left(a_{1} A_{1}+a_{2} A_{2}+a_{3} A_{3}\right)+10 a_{1} a_{2} A_{1}\right],
$$

$$
\begin{aligned}
C_{21}=\frac{7}{6} & {\left[2 x_{01}\left(A_{1} x_{01}+A_{2} x_{02}+A_{3} x_{03}\right)\right.} \\
& \left.+A_{1}\left(x_{01}^{2}-4 x_{02}^{2}+x_{03}^{2}\right)-10 A_{2} x_{01} x_{02}\right] \\
& +\frac{14}{15}\left[x_{01}\left(-3 B_{1}+4 B_{2}-B_{3}\right)+4 B_{12} x_{02}-B_{31} x_{03}\right]
\end{aligned}
$$

$$
\begin{aligned}
+ & \frac{7}{18}\left[A_{1}\left(-a_{1}^{2}+4 a_{2}^{2}-a_{3}^{2}\right)\right. \\
& \left.-2 a_{1}\left(a_{1} A_{1}+a_{2} A_{2}+a_{3} A_{3}\right)+10 a_{1} a_{2} A_{2}\right] \\
C_{13}=\frac{7}{6}\left[2 x_{03}\left(A_{1} x_{01}+A_{2} x_{02}+A_{3} x_{03}\right)\right. & \left.-10 A_{1} x_{01} x_{03}+A_{3}\left(-4 x_{01}^{2}+x_{02}^{2}+x_{03}^{2}\right)\right] \\
+ & \frac{14}{15}\left[x_{03}\left(4 B_{1}-B_{2}-3 B_{3}\right)-B_{23} x_{02}+4 B_{31} x_{01}\right] \\
+ & \frac{7}{18}\left[A_{3}\left(4 a_{1}^{2}-a_{2}^{2}-a_{3}^{2}\right)\right. \\
& \left.\quad-2 a_{3}\left(a_{1} A_{1}+a_{2} A_{2}+a_{3} A_{3}\right)+10 a_{1} a_{3} A_{1}\right] \\
+ & \frac{35}{9}\left(a_{1} a_{2} A_{3}+a_{2} a_{3} A_{1}+a_{3} a_{1} A_{2}\right) \\
+ & \frac{14}{3}\left(B_{12} x_{03}+B_{23} x_{01}+B_{31} x_{02}\right) \\
& \left.+A_{3} x_{01} x_{02}+A_{2} x_{01} x_{03}+A_{1} x_{02} x_{03}\right) \\
+ &
\end{aligned}
$$

Introducing the quantities

$$
\begin{aligned}
D_{1}=\frac{18 C_{1}}{7}-6( & -A_{1} x_{01}^{2}+A_{2} x_{01} x_{02}+A_{3} x_{01} x_{03} \\
& +\frac{A_{1} x_{02}^{2}}{2}+\frac{A_{1} x_{03}^{2}}{2} \\
& \left.+\frac{6 B_{1} x_{01}}{5}-\frac{2 B_{12} x_{02}}{5}-\frac{2 B_{31} x_{03}}{5}\right), \\
D_{2}=\frac{18 C_{2}}{7}-6\left(A_{1} x_{01} x_{02}-A_{2} x_{02}^{2}+A_{3} x_{02} x_{03}\right. & \\
& +\frac{A_{2} x_{01}^{2}}{2}+\frac{A_{2} x_{03}^{2}}{2} \\
& \left.-\frac{2 B_{12} x_{01}}{5}+\frac{6 B_{2} x_{02}}{5}-\frac{2 B_{23} x_{03}}{5}\right),
\end{aligned}
$$

$$
\begin{aligned}
D_{3}=\frac{18 C_{3}}{7}-6( & A_{1} x_{01} x_{03}+A_{2} x_{02} x_{03}-A_{3} x_{03}^{2} \\
& +\frac{A_{3} x_{01}^{2}}{2}+\frac{A_{3} x_{02}^{2}}{2} \\
& \left.-\frac{2 B_{31} x_{01}}{5}-\frac{2 B_{23} x_{02}}{5}+\frac{6 B_{3} x_{03}}{5}\right),
\end{aligned}
$$




$$
\begin{aligned}
& D_{4}=\frac{18 C_{12}}{7}-6\left(-4 A_{1} x_{01} x_{02}-2 A_{2} x_{01}^{2}+A_{3} x_{02} x_{03}\right. \\
& +\frac{3 A_{2} x_{02}^{2}}{2}+\frac{A_{2} x_{03}^{2}}{2} \\
& +\frac{2}{5} x_{02}\left(4 B_{1}-3 B_{2}-B_{3}\right) \\
& \left.+\frac{8 B_{12} x_{01}}{5}-\frac{2 B_{23} x_{03}}{5}\right) \\
& D_{5}=\frac{18 C_{21}}{7}-6\left(-4 A_{2} x_{01} x_{02}-2 A_{1} x_{02}^{2}+A_{3} x_{01} x_{03}\right. \\
& +\frac{3 A_{1} x_{01}^{2}}{2}+\frac{A_{1} x_{03}^{2}}{2} \\
& +\frac{2}{5} x_{01}\left(-3 B_{1}+4 B_{2}-B_{3}\right) \\
& \left.+\frac{8 B_{12} x_{02}}{5}-\frac{2 B_{31} x_{03}}{5}\right) \\
& D_{6}=\frac{18 C_{13}}{7}-6\left(-4 A_{1} x_{01} x_{03}+A_{2} x_{02} x_{03}-2 A_{3} x_{01}^{2}\right. \\
& +\frac{A_{3} x_{02}^{2}}{2}+\frac{3 A_{3} x_{03}^{2}}{2} \\
& +\frac{2}{5} x_{03}\left(4 B_{1}-B_{2}-3 B_{3}\right) \\
& \left.+\frac{8 B_{31} x_{01}}{5}-\frac{2 B_{23} x_{02}}{5}\right), \\
& D_{10}=\frac{18 C_{123}}{7}-6\left(-5 A_{3} x_{01} x_{02}\right. \\
& -5 A_{1} x_{02} x_{03}-5 A_{2} x_{01} x_{03} \\
& \left.+2 B_{23} x_{01}+2 B_{31} x_{02}+2 B_{12} x_{03}\right) \text {, }
\end{aligned}
$$

the system is further reduced to

$$
\begin{aligned}
& D_{1}=A_{1}\left(4 a_{1}^{2}-a_{2}^{2}-a_{3}^{2}\right)-2 a_{1}\left(a_{1} A_{1}+a_{2} A_{2}+a_{3} A_{3}\right), \\
& D_{2}=A_{2}\left(-a_{1}^{2}+4 a_{2}^{2}-a_{3}^{2}\right)-2 a_{2}\left(a_{1} A_{1}+a_{2} A_{2}+a_{3} A_{3}\right), \\
& D_{3}=A_{3}\left(-a_{1}^{2}-a_{2}^{2}+4 a_{3}^{2}\right)-2 a_{3}\left(a_{1} A_{1}+a_{2} A_{2}+a_{3} A_{3}\right), \\
& D_{4}=A_{2}\left(4 a_{1}^{2}-a_{2}^{2}-a_{3}^{2}\right)-2 a_{2}\left(-4 a_{1} A_{1}+a_{2} A_{2}+a_{3} A_{3}\right), \\
& D_{5}=A_{1}\left(-a_{1}^{2}+4 a_{2}^{2}-a_{3}^{2}\right)-2 a_{1}\left(a_{1} A_{1}-4 a_{2} A_{2}+a_{3} A_{3}\right), \\
& D_{6}=A_{3}\left(4 a_{1}^{2}-a_{2}^{2}-a_{3}^{2}\right)-2 a_{3}\left(-4 a_{1} A_{1}+a_{2} A_{2}+a_{3} A_{3}\right), \\
& D_{10}=10\left(a_{1} a_{2} A_{3}+A_{1} a_{2} a_{3}+A_{2} a_{1} a_{3}\right) .
\end{aligned}
$$

With the help of a series of appropriate row operations we can rewrite the system (55) in the form

$$
\begin{aligned}
0= & -2\left(a_{1} a_{2}\right)^{2}\left(a_{2} a_{3}\right)\left(A_{1}^{2}+2 A_{2}^{2}\right) \\
& +3 A_{1} A_{2}\left[\left(a_{1} a_{2}\right)^{2}\left(a_{1} a_{3}\right)-\left(a_{1} a_{3}\right)\left(a_{2} a_{3}\right)^{2}\right] \\
& -\left(a_{1} a_{2}\right)\left(a_{2} a_{3}\right)\left(A_{1} D_{2}+2 A_{2} D_{1}\right) \\
& -2 A_{1}\left(a_{1} a_{2}\right)\left(a_{2} a_{3}\right)^{2} A_{3}-4\left(a_{1} a_{2}\right)\left(a_{2} a_{3}\right)\left(a_{1} a_{3}\right) A_{2} A_{3},
\end{aligned}
$$

$$
\begin{gathered}
0=-2\left(a_{1} a_{2}\right)^{2}\left(a_{1} a_{3}\right)\left(2 A_{1}^{2}+A_{2}^{2}\right) \\
+3 A_{1} A_{2}\left[\left(a_{1} a_{2}\right)^{2}\left(a_{2} a_{3}\right)-\left(a_{1} a_{3}\right)^{2}\left(a_{2} a_{3}\right)\right] \\
-\left(a_{1} a_{2}\right)\left(a_{1} a_{3}\right)\left(2 A_{1} D_{2}+A_{2} D_{1}\right) \\
-4 A_{1}\left(a_{1} a_{2}\right)\left(a_{1} a_{3}\right)\left(a_{2} a_{3}\right) A_{3}-2\left(a_{1} a_{2}\right)\left(a_{1} a_{3}\right)^{2} A_{2} A_{3}, \\
a_{2} a_{3}=\frac{1}{10 A_{3}\left(A_{1}^{2}+A_{2}^{2}\right)\left(A_{1}^{2}+A_{2}^{2}+2 A_{3}^{2}\right)} \\
\times\left(-4 A_{1}^{4} D_{2}-A_{1}^{4} D_{4}+3 A_{1}^{3} A_{2} D_{1}+2 A_{1}^{3} A_{2} D_{5}\right. \\
\quad-3 A_{1}^{2} A_{2}^{2} D_{2}-2 A_{1}^{2} A_{2}^{2} D_{4}-5 A_{1}^{2} A_{2} A_{3} D_{3} \\
\quad-8 A_{1}^{2} A_{3}^{2} D_{2}-2 A_{1}^{2} A_{3}^{2} D_{4}+4 A_{1} A_{2}^{3} D_{1} \\
+A_{1} A_{2}^{3} D_{5}-A_{1} A_{2} A_{3}^{2} D_{1}+A_{1} A_{2} A_{3}^{2} D_{5} \\
\left.-5 A_{2}^{3} A_{3} D_{3}-7 A_{2}^{2} A_{3}^{2} D_{2}-3 A_{2}^{2} A_{3}^{2} D_{4}\right)
\end{gathered}
$$

$$
\begin{aligned}
a_{1} a_{3}= & \frac{1}{10 A_{3}\left(A_{1}^{2}+A_{2}^{2}\right)\left(A_{1}^{2}+A_{2}^{2}+2 A_{3}^{2}\right)} \\
& \times\left(4 A_{1}^{3} A_{2} D_{2}+A_{1}^{3} A_{2} D_{4}-5 A_{1}^{3} A_{3} D_{3}-3 A_{1}^{2} A_{2}^{2} D_{1}\right. \\
& -2 A_{1}^{2} A_{2}^{2} D_{5}-7 A_{1}^{2} A_{3}^{2} D_{1}-3 A_{1}^{2} A_{3}^{2} D_{5} \\
& +3 A_{1} A_{2}^{3} D_{2}+2 A_{1} A_{2}^{3} D_{4}-5 A_{1} A_{2}^{2} A_{3} D_{3} \\
& -A_{1} A_{2} A_{3}^{2} D_{2}+A_{1} A_{2} A_{3}^{2} D_{4}-4 A_{2}^{4} D_{1}-A_{2}^{4} D_{5} \\
& \left.-8 A_{2}^{2} A_{3}^{2} D_{1}-2 A_{2}^{2} A_{3}^{2} D_{5}\right)
\end{aligned}
$$$$
0=\left(a_{1} a_{2}\right)\left(-4 A_{1}^{2} A_{3}-14 A_{2}^{2} A_{3}\right)
$$$$
+\left(a_{1} a_{3}\right)\left(-24 A_{1}^{2} A_{2}-14 A_{2} A_{3}^{2}\right)
$$$$
+\left(a_{2} a_{3}\right)\left(6 A_{1} A_{2}^{2}-4 A_{1} A_{3}^{2}\right)
$$$$
+3 A_{1} A_{2} D_{6}-2 A_{1} A_{3} D_{2}-7 A_{2} A_{3} D_{1} \text {, }
$$ 


$$
0=10 A_{1}\left(a_{2} a_{3}\right)+10\left(a_{1} a_{2}\right) A_{3}+10\left(a_{1} a_{3}\right) A_{2}-D_{10} .
$$

Inserting (58), (59), and (60) that provide the values of the products $a_{1} a_{2}, a_{2} a_{3}$, and $a_{3} a_{1}$, respectively, in the remaining four equations of the system and inserting the expressions for the $D$ 's given in (55) we arrive at seven equations of the form

$$
\begin{aligned}
0 & =P_{1}\left(x_{01}, x_{02}, x_{03}\right), \\
0 & =P_{2}\left(x_{01}, x_{02}, x_{03}\right), \\
a_{1} a_{2} & =P_{3}\left(x_{01}, x_{02}, x_{03}\right), \\
a_{2} a_{3} & =P_{4}\left(x_{01}, x_{02}, x_{03}\right), \\
a_{3} a_{1} & =P_{5}\left(x_{01}, x_{02}, x_{03}\right), \\
0 & =P_{6}\left(x_{01}, x_{02}, x_{03}\right), \\
0 & =P_{7}\left(x_{01}, x_{02}, x_{03}\right),
\end{aligned}
$$

where the functions $P_{1}$ and $P_{2}$ are polynomials of degree five in the variables $x_{01}, x_{02}$, and $x_{03}$, while the functions $P_{6}$ and $P_{7}$ are polynomials of the first degree in the same variables. Solving the system (68), (69) with respect to $x_{02}$ and $x_{03}$ in terms of $x_{01}$ and substituting these values in either of the functions $P_{1}$ or $P_{2}$ we end up with a fifth degree polynomial in the variable $x_{01}$ which can be solved easily with a standard numerical scheme. Once $x_{01}$ is obtained we calculate via substitution of the values of $x_{02}, x_{03}$ and $a_{1}, a_{2}, a_{3}$. Some further trivial substitutions lead to the solution of the inverse problem.

The following numerical example demonstrates the validity of this hybrid inversion algorithm.

From the solution of the corresponding forward problem, with

$$
\begin{aligned}
\left(Q_{1}, Q_{2}, Q_{3}\right) & =\left(\frac{3}{12}, \frac{4}{12}, \frac{2}{12}\right), \\
\left(x_{01}, x_{02}, x_{03}\right) & =(1,2,-2), \\
\left(\alpha_{1}, \alpha_{2}, \alpha_{3}\right) & =\left(\frac{2}{3}, \frac{2}{3}, \frac{1}{3}\right)
\end{aligned}
$$

and $L=2$, we extract the following values of the $A, B$, and $C$ constants:

$$
\begin{gathered}
\left(A_{1}, A_{2}, A_{3}\right)=(3.0453463,4.0275938,1.9948525), \\
\left(B_{1}, B_{2}, B_{3}\right)=(12.301466,13.710550,-26.012016), \\
\left(B_{12}, B_{23}, B_{31}\right)=(51.666667,-10.3150859,-4.8332202), \\
\left(C_{1}, C_{2}, C_{3}\right)=(-45.587908,-12.703180,103.68997), \\
\left(C_{12}, C_{21}, C_{13}\right)=(235.73554,323.78438,-154.79058), \\
C_{123}=-347.68131
\end{gathered}
$$

which imply that (68) and (69) assume the form

$$
\begin{aligned}
& 0=130.352+3.94029 x_{01}-24.2337 x_{02}+42.9124 x_{03}, \\
& 0=-2.88367-56.3856 x_{01}+38.3283 x_{02}+8.69373 x_{03} .
\end{aligned}
$$

Hence, we obtain the expressions

$$
\begin{aligned}
& x_{01}=4.05320+1.52660 x_{03}, \\
& x_{02}=6.03799+2.01899 x_{03} .
\end{aligned}
$$

Inserting these values of $x_{01}, x_{02}$ in (63)-(67) we arrive at the following system:

$$
\begin{gathered}
0=-61.2662+137.598 x_{03}+378.011 x_{03}^{2} \\
+282.942 x_{03}^{3}+87.3784 x_{03}^{4}+9.69061 x_{03}^{5}, \\
0=-150.468-460.991 x_{03}-513.007 x_{03}^{2} \\
-264.509 x_{03}^{3}-63.2272 x_{03}^{4}-5.50254 x_{03}^{5}, \\
a_{1} a_{2}=7.03737+21.123 x_{03}+9.2466 x_{03}^{2}, \\
a_{2} a_{3}=25.2989+24.319 x_{03}+6.05698 x_{03}^{2}, \\
a_{1} a_{3}=12.7037+15.067 x_{03}+4.57981 x_{03}^{2}, \\
x_{01}=4.05320+1.52660 x_{03}, \\
x_{02}=6.03799+2.01899 x_{03} .
\end{gathered}
$$

The numerical solution of (75) yields the five approximate roots

$$
\begin{aligned}
& x_{31}=-4.89255, \\
& x_{32}=-2.00021, \\
& x_{33}=-1.99979, \\
& x_{34}=-1.83761, \\
& x_{35}=-0.760384 .
\end{aligned}
$$

However, only the roots

$$
\begin{aligned}
& x_{32}=-2.00021, \\
& x_{33}=-1.99979
\end{aligned}
$$

which are very close to the value -2 satisfy (74) as well. In fact, eliminating the fifth degree terms among (74) and (75) we obtain the fourth degree equation

$$
\begin{aligned}
-P_{1}-\frac{9.69061}{5.50254} P_{2}= & 23.972 x_{03}^{4}+182.888 x_{03}^{3} \\
& +525.453 x_{03}^{2}+674.259 x_{03}+326.258 \\
= & 0
\end{aligned}
$$


which has the double real root $x_{03}=-2$. Then, by a straightforward substitution of $x_{32}$ and $x_{33}$ we obtain

$$
\begin{gathered}
\left(x_{01}, x_{02}, x_{03}, a_{1} a_{2}, a_{2} a_{3}, a_{1} a_{3}\right)_{1} \\
=(0.999677,1.99957,-2.00021, \\
1.78113,0.888872,0.889575), \\
\left(x_{01}, x_{02}, x_{03}, a_{1} a_{2}, a_{2} a_{3}, a_{1} a_{3}\right)_{2} \\
=(1.00032,2.00042,-1.99979, \\
1.77444,0.888910,0.888205)
\end{gathered}
$$

or

$$
\begin{gathered}
\left(x_{01}, x_{02}, x_{03}, a_{1}, a_{2}, a_{3}\right)_{1} \\
=(0.999677,1.99957,-2.00021, \\
1.33512,1.33406,0.666290), \\
\left(x_{01}, x_{02}, x_{03}, a_{1}, a_{2}, a_{3}\right)_{2} \\
=(0.999677,1.99957,-2.00021, \\
\quad-1.33512,-1.33406,-0.666290), \\
\left(x_{01}, x_{02}, x_{03}, a_{1}, a_{3}, a_{3}\right)_{3} \\
=(1.00032,2.00042,-1.99979, \\
1.33155,1.33261,0.667044), \\
\left(x_{01}, x_{02}, x_{03}, a_{1}, a_{3}, a_{3}\right)_{4}
\end{gathered}
$$$$
\text { (1.00032, 2.00042, -1.99979, }
$$$$
-1.33155,-1.33261,-0.667044) \text {. }
$$

For comparison we also give the corresponding approximate solution, obtained by the function NSolve of Mathematica, for the $6 \times 6$ system (47), (48), (50)-(53), which is

$$
\begin{aligned}
&\left(x_{01}, x_{02}, x_{03}, a_{1}, a_{2}, a_{3}\right)_{1} \\
&=(1.00000,2.00000,-2.00000, \\
&1.33333,1.33333,0.666666), \\
&\left(x_{01}, x_{02}, x_{03}, a_{1}, a_{3}, a_{3}\right)_{2} \\
&=(1.00000,2.00000,-2.00000,-1.33333, \\
&\quad-1.33333,-0.666666), \\
&\left(x_{01}, x_{02}, x_{03}, a_{1}, a_{2}, a_{3}\right)_{3}(1.00000,2.00000,-2.00000,1.33333, \\
&1.33333,0.666666), \\
&\left(x_{01}, x_{02}, x_{03}, a_{1}, a_{3}, a_{3}\right)_{4} \\
&=(1.00000,2.00000,-2.00000,-1.33333, \\
&\quad-1.33333,-0.666666),
\end{aligned}
$$

$$
\begin{aligned}
& \left(x_{01}, x_{02}, x_{03}, a_{1}, a_{2}, a_{3}\right)_{5} \\
& =(-3.82890,-10.3370,-7.00206 \text {, } \\
& 11.9783,22.5330,7.88115) \text {, } \\
& \left(x_{01}, x_{02}, x_{03}, a_{1}, a_{2}, a_{3}\right)_{6} \\
& =(-3.82890,-10.3370,-7.00206 \text {, } \\
& -11.9783,-22.5330,-7.88115) \text {, } \\
& \left(x_{01}, x_{02}, x_{03}, a_{1}, a_{3}, a_{3}\right)_{7} \\
& =(7.12154,0.778341,-0.869492 \text {, } \\
& \text { 6.09759, -3.74636, 4.17589), } \\
& \left(x_{01}, x_{02}, x_{03}, a_{1}, a_{3}, a_{3}\right)_{8} \\
& =(7.12154,0.778341,-0.869492 \text {, } \\
& -6.09759,3.74636,-4.17589) \text {. }
\end{aligned}
$$

Among these eight solutions, only the following four are compatible with (49):

$$
\begin{aligned}
\left(x_{01}, x_{02}, x_{03}, a_{1}, a_{2}, a_{3}\right)_{1} & \\
= & \left(x_{01}, x_{02}, x_{03}, a_{1}, a_{2}, a_{3}\right)_{3} \\
= & (1.00000,2.00000,-2.00000,1.33333, \\
& 1.33333,0.666666), \\
\left(x_{01}, x_{02}, x_{03}, a_{1}, a_{3}, a_{3}\right)_{2} & \left(x_{01}, x_{02}, x_{03}, a_{1}, a_{2}, a_{3}\right)_{4} \\
= & (1.00000,2.00000,-2.00000,-1.33333, \\
& \quad-1.33333,-0.666666) .
\end{aligned}
$$

These two solutions are compatible with the initial system (31)-(34).

These two solutions are physically equivalent since they identify the same position $\left(x_{01}, x_{02}, x_{03}\right)$ and two opposite directions $\pm\left(a_{1}, a_{2}, a_{3}\right)$ identifying the line segment that supports the current.

Consequently, the initial system has a unique solution which implies that the one-dimensional current distribution is fully recoverable from EEG data.

As an analytical application we solve, in the sequel, the system in two special cases based on some a priori information about the characteristics of the current.

Special Case I. Let us assume that we have the a priori information that the line segment is oriented along the $x_{1}$ axis and that its middle point is $\mathbf{r}_{0}=\left(0,0, r_{0}\right)$. Then, from (44) 
we obtain the distance of the middle point from the center of the sphere

$$
r_{0}=\frac{2}{5} \frac{B_{23}}{A_{2}},
$$

from (38) we obtain the semilength of the line segment

$$
L=\sqrt{\frac{9}{14} \frac{C_{12}}{A_{2}}+\frac{3}{25}\left(\frac{B_{23}}{A_{2}}\right)^{2}},
$$

and finally, from (30) we obtain the moment

$$
\mathbf{Q}=\frac{1}{6}\left[\frac{9}{14} \frac{C_{12}}{A_{2}}+\frac{3}{25}\left(\frac{B_{23}}{A_{2}}\right)^{2}\right]^{-1 / 2} \mathbf{A} .
$$

Special Case II. If the $\mathbf{r}_{0}=\left(0,0, r_{0}\right)$ is located at the same point as in the previous case but now the segment is oriented along the $x_{3}$-axis, then from (41), (42) we obtain

$$
r_{0}=\frac{15}{56} \frac{A_{2} C_{31}-A_{1} C_{32}}{A_{2} B_{31}-A_{1} B_{32}}
$$

and from (35) we obtain

$$
\begin{aligned}
L=\left(3\left(\frac{15}{56} \frac{A_{2} C_{31}-A_{1} C_{32}}{A_{2} B_{31}-A_{1} B_{32}}\right)^{2}\right. & \\
& \left.-\frac{9}{14} \frac{B_{31}}{A_{1}} \frac{A_{2} C_{31}-A_{1} C_{32}}{A_{2} B_{31}-A_{1} B_{32}}-\frac{18}{7} \frac{C_{1}}{A_{1}}\right)^{1 / 2} .
\end{aligned}
$$

As before, the moment is obtained from (30) and it is equal to

$$
\begin{aligned}
\mathbf{Q}=\frac{1}{6} & {\left[3\left(\frac{15}{56} \frac{A_{2} C_{31}-A_{1} C_{32}}{A_{2} B_{31}-A_{1} B_{32}}\right)^{2}\right.} \\
& \left.-\frac{9}{14} \frac{B_{31}}{A_{1}} \frac{A_{2} C_{31}-A_{1} C_{32}}{A_{2} B_{31}-A_{1} B_{32}}-\frac{18}{7} \frac{C_{1}}{A_{1}}\right]^{-1 / 2} \mathbf{A} .
\end{aligned}
$$

\section{Conflict of Interests}

The authors declare that there is no conflict of interests regarding the publication of this paper.

\section{Acknowledgments}

The present work is part of the project "Functional Brain," which is implemented within the "ARISTEIA" Action of the "OPERATIONAL PROGRAMME EDUCATION AND LIFELONG LEARNING" and is cofunded by the European Social Fund (ESF) and National Resources.

\section{References}

[1] J. Malmivuo and R. Plonsey, Bioelectromagnetism, Oxford University Press, New York, NY, USA, 1995.

[2] L. D. Landau and E. M. Lifshitz, Electrodynamics of Continuous Media, Pergamon Press, London, UK, 1960.
[3] R. Plonsey and D. B. Heppner, "Considerations of quasistationarity in electrophysiological systems," The Bulletin of Mathematical Biophysics, vol. 29, no. 4, pp. 657-664, 1967.

[4] S. He and V. G. Romanov, "Identification of dipole sources in a bounded domain for Maxwell's equations," Wave Motion, vol. 28, no. 1, pp. 25-40, 1998.

[5] G. Dassios, "Electric and magnetic activity of the brain in spherical and ellipsoidal geometry," in Mathematical Modeling in Biomedical Imaging, H. Ammari, Ed., vol. 183 of Mathematical Biosciences Subseries, pp. 133-202, Springer, Berlin, Germany, 2009.

[6] G. Dassios and A. S. Fokas, "The definite non-uniqueness results for deterministic EEG and MEG data," Inverse Problems, vol. 29, no. 6, Article ID 065012, 10 pages, 2013.

[7] H. Helmholtz, "Ueber einige Gesetze der Vertheilung elektrischer Ströme in körperlichen Leitern mit Anwendung auf die thierisch-elektrischen Versuche," Annalen der Physik und Chemie, vol. 89, pp. 211-233, 353-377, 1853.

[8] M. Hamalainen, R. Hari, R. J. Ilmoniemi, J. Knuutila, and O. V. Lounasmaa, "Magnetoencephalography-theory, instrumentation, and applications to noninvasive studies of the working human brain," Reviews of Modern Physics, vol. 65, no. 2, pp. 413497, 1993.

[9] H. Ammari, G. Bao, and J. L. Fleming, "An inverse source problem for Maxwell's equations in magnetoencephalography," SIAM Journal on Applied Mathematics, vol. 62, no. 4, pp. 13691382, 2002.

[10] R. Albanese and P. B. Monk, "The inverse source problem for Maxwell's equations," Inverse Problems, vol. 22, no. 3, pp. 10231035, 2006.

[11] G. Dassios and A. S. Fokas, "Electro-magneto-encephalography for a three-shell model: dipoles and beyond for the spherical geometry," Inverse Problems, vol. 25, no. 3, Article ID 035001, 20 pages, 2009.

[12] G. Dassios, Ellipsoidal Harmonics: Theory and Applications, vol. 146 of Encyclopedia of Mathematics and Its Applications, Cambridge University Press, Cambridge, Mass, USA, 2012.

[13] P. M. Morse and H. Feshbach, Methods of Theoretical Physics, vol. 2, McGraw-Hill, New York, NY, USA, 1st edition, 1953.

[14] P. M. Morse and H. Feshbach, Methods of Theoretical Physics, vol. 1, McGraw-Hill, New York, NY, USA, 1st edition, 1953.

[15] G. Dassios and A. S. Fokas, "Electro-magneto-encephalography and fundamental solutions," Quarterly of Applied Mathematics, vol. 67, no. 4, pp. 771-780, 2009.

[16] L. Brand, Vector and Tensor Analysis, John Wiley \& Sons, New York, NY, USA, 1st edition, 1947. 


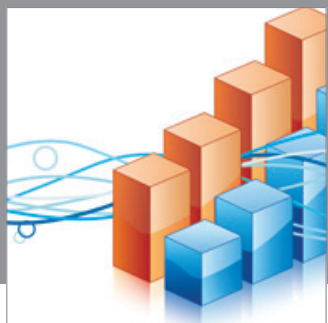

Advances in

Operations Research

mansans

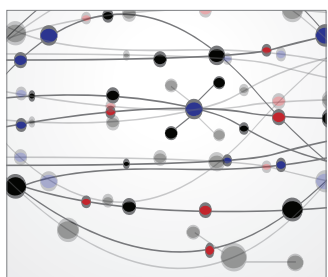

The Scientific World Journal
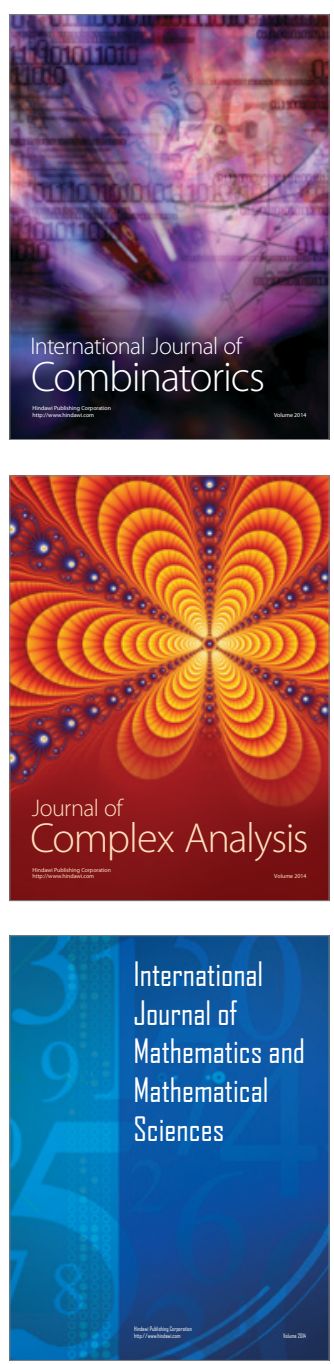
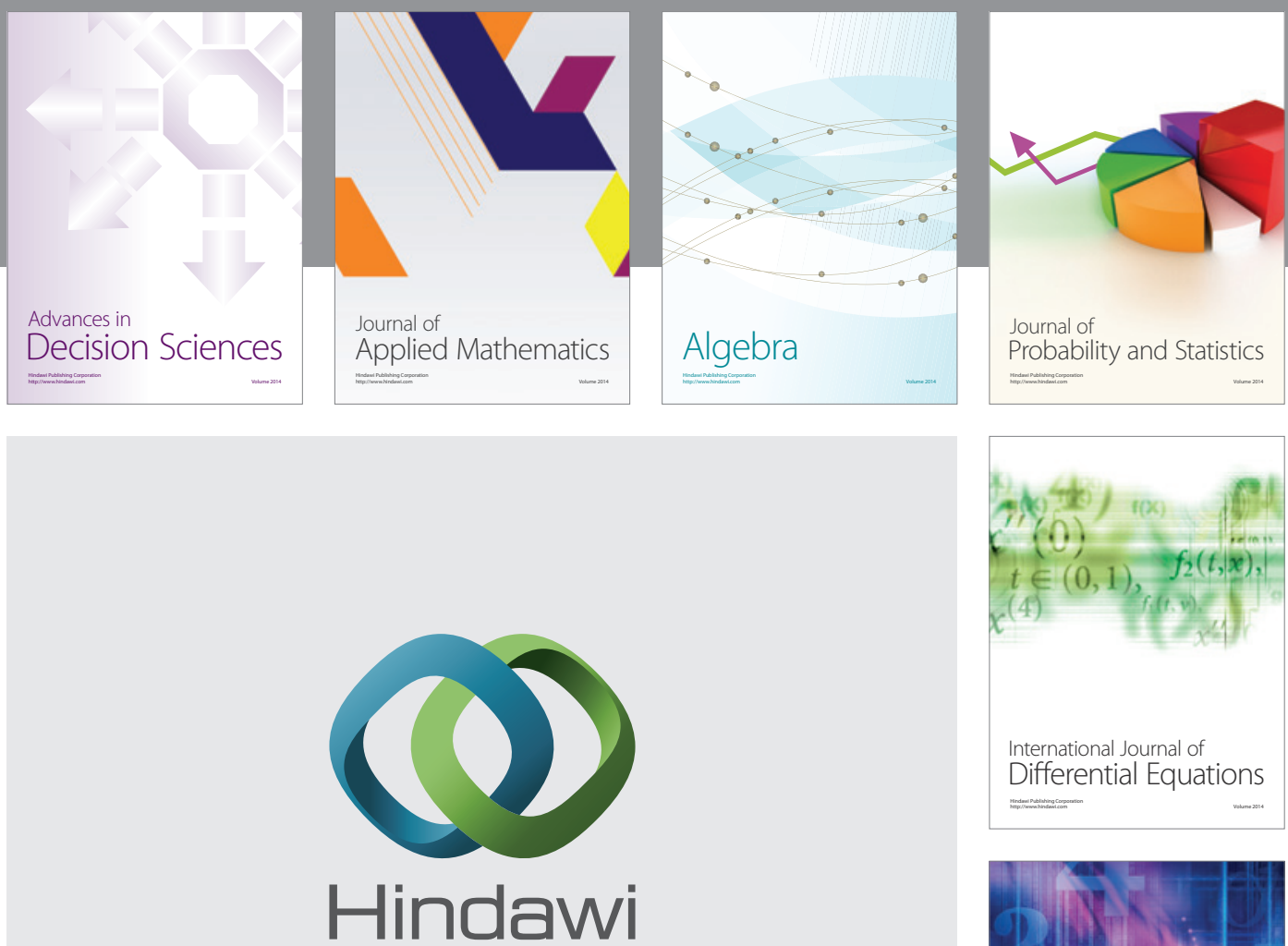

Submit your manuscripts at http://www.hindawi.com
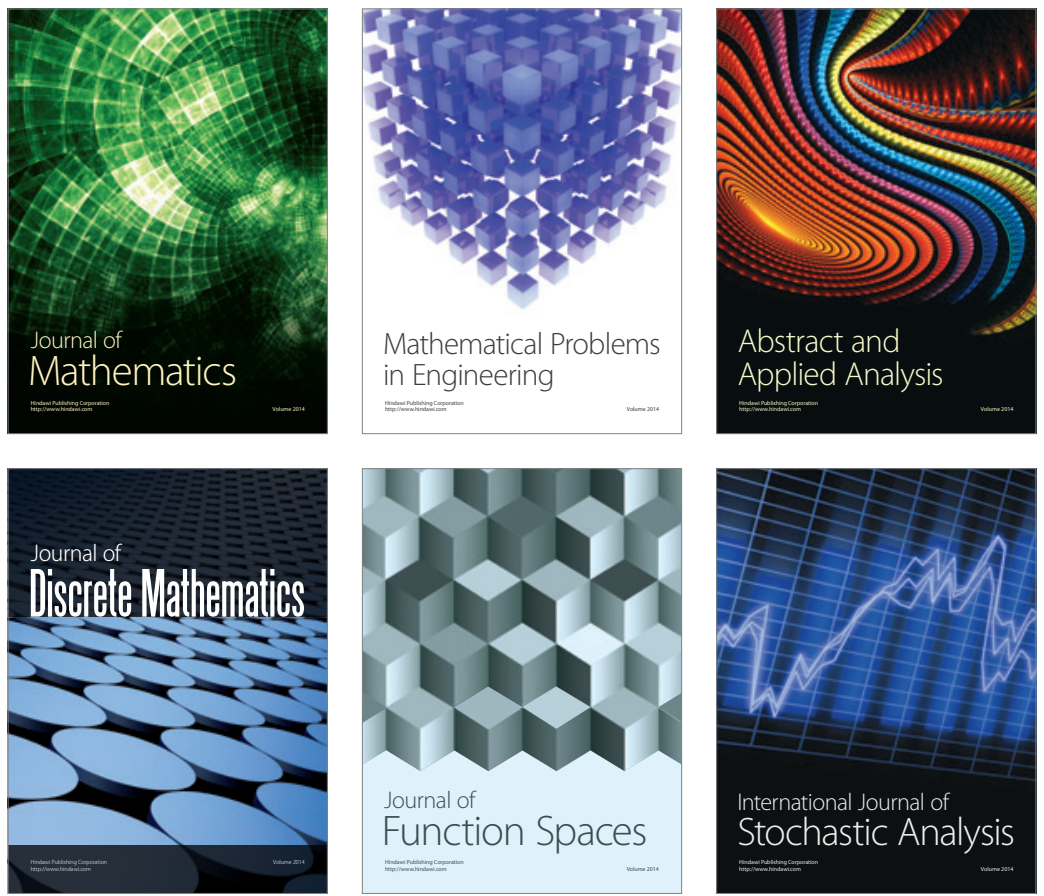

Journal of

Function Spaces

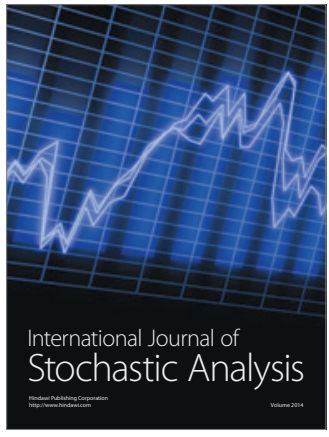

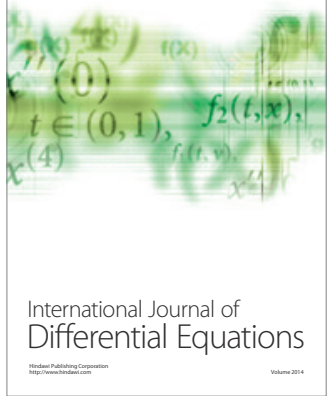
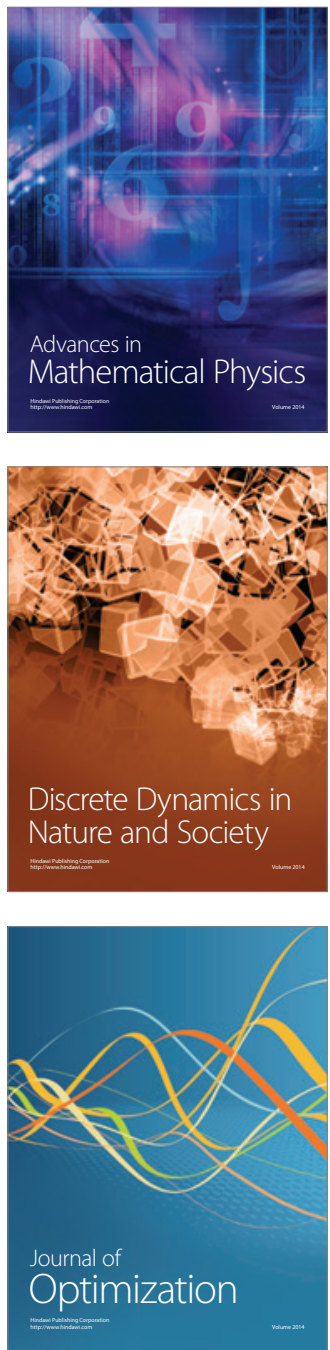